\title{
REVIEWS
}

\section{Burning Mouth Syndrome}

\section{David Mock*, Deepika Chugh}

Faculty of Dentistry, University of Toronto, Wasser Pain Management Centre, Mount Sinai Hospital, Toronto, Canada

\begin{abstract}
Most clinicians dread seeing the patient presenting with a primary complaint of a burning pain on one or more oral mucosal surfaces. Unlike most other clinical conditions presenting in a dental office, burning mouth syndrome is poorly understood with few evidence based remedies. More
\end{abstract}

recently, advances have been made towards clarifying the possible etiology of the disorder and testing the possible therapeutic modalities available. This article attempts to summarize the "state of the art" today.

Keywords orofacial pain, burning mouth syndrome (BMS), stomatodynia, neuropathic pain

Received Jan. 4, 2010; Revision accepted Feb. 24, 2010

\section{Introduction}

The patient with a complaint of a burning sensation of the oral mucosa presents one of the most difficult challenges to the health care professional. There are a variety of names applied to this presentation including, but not limited to, burning mouth syndrome (the most widely accepted), stomatodynia, stomatopyrosis, glossopyrosis, glossodynia, sore mouth, sore tongue and oral dysesthesia. The Headache Classification Subcommittee of the International Headache Society defines this condition as "an intraoral burning sensation for which no medical or dental cause can be found" (Headache Classification Committee of the International Headache Society, 2004). The diagnosis is therefore one of exclusion and thus often not particularly satisfying to patient or doctor alike.

\section{Clinical Presentation}

The patient will present complaining burning pain involving the oral mucosa, most often the tongue, hard palate and/or lips. Usually the onset is spontaneous but at times there is a precipitating event such as trauma or dental treatment. There can be an associated xerostomia (or the sensation thereof), dysesthesia and/or dysgeusia. A poor quality of life, depression, anxiety and/or somatization are also often associated with this disorder. It is considerably more common in women than men (33:1) and most often perimenopausal or postmenopausal women (Lipton et al., 1993). Burning mouth also often coexists with other chronic pain disorders (Wessely et al., 1999; Zakrzewska, 1995).

\section{Differential Diagnosis}

As already noted, the diagnosis of burning mouth syndrome depends on exclusion of a detectable organic basis for the complaint. Therefore a number of local conditions must be considered and ruled out first.

The first and commonest cause is local irritation. A rough prosthesis or dental restoration will irritate the tongue and cheeks. Contact hypersensitivity to dental materials has been suggested as one possible 
mechanism although this has not been confirmed with patch testing (Marino et al., 2009). Caustic oral rinses or acidic foods can cause generalized oral mucosal irritation and hypersensitivity. Similarly, tongue or cheek biting habits will result in localized discomfort that can, on occasion, present as a burning sensation as can smoking (Gao et al., 2009).

Oral candidiasis is not an uncommon condition in this patient population. This can be a primary infection but most often is secondary to either local irritation or systemic predisposition.

Although oral candidiasis most often presents as a white, red or mixed red/white lesion, sometimes there is little visible change. A direct smear is the ideal way to make this diagnosis rather than taking samples for microbiological culture as the organism, Candida albicans is commensal in most mouths. If candidiasis is suspected, a course of antifungal therapy, such as mycostatin, should be tried first.

Various mucocutaneous diseases/disorders also need to be considered. These include lichen planus, lichenoid reactions, benign mucous membrane pemphigoid, pemphigus and migratory glossitis. A visual clinical examination, possibly followed by biopsy will confirm or exclude these diagnoses. Similarly, viral diseases such as herpes simplex or zoster can result in symptoms that the patient interprets as a burning sensation. Although these will be clinically apparent when the lesions erupt, there can be a prodromal burning sensation. Although the pain of post herpetic neuralgia is usually much more severe, occasionally it can also present as a burning sensation on the oral mucosa.

Nutritional, metabolic or endocrine disorders may also result in a burning mouth. This includes diabetes, hypothyroidism, iron or zinc deficiency and vitamin $\mathrm{B}$ complex deficiency, particularly vitamin $B_{12}$ (cobalamin). Vitamin $B_{12}$ deficiency is a common cause of macrocytic anemia (pernicious anemia). Serum vitamin $B_{12}$ levels will only identify $50 \%$ of patients with subclinical disease and therefore measurement of serum methylmalonic acid and homocysteine levels are recommended as a more sensitive method of screening for vitamin $\mathrm{B}_{12}$ deficiency (Oh and Brown, 2003). These are increase much earlier in a case of vitamin $B_{12}$ deficiency.

Finally, patients with true xerostomia will complain of a burning mouth. This can be as a component of Sjögren's syndrome, a result of radiation therapy to the head and neck, a side effect of medication or just an age related decrease in salivary production, particularly the serous component.

Thus it behooves the clinician to obtain a clear and detailed illness and medical/dental history as well as perform a thorough oral clinical examination including any laboratory studies indicated. A neurological examination can be useful although, unless there are marked deficiencies, the lack of baseline data can present a problem.

If other causes of this symptom are ruled out and/or the patient fails to respond to a normal course of treatment a diagnosis of BMS is reasonable.

\section{Pathogenesis}

It would be foolhardy to not start with the statement that the etiology and pathogenesis of burning mouth syndrome is unknown. In fact, there is ongoing debate as to whether it is primarily a physiological or a somatoform disorder. At present, most accept that the etiology is multifactorial with mounting evidence for a physiological basis. Axonal degenerative changes have been demonstrated in glossal terminal nerve fibres and sensory changes have been shown to be present in burning mouth patients, particularly perception of heat, cold, taste and nocioceptive stimuli (Formaker et al., 1998; Grémeau-Richard et al., 2004; Yilmaz et al., 2007). Abnormalities in trigeminal somatosensory evoked potentials have been demonstrated as well (Gao et al., 2000). This, plus other data, strongly suggests that there is a dysfunction of the small diameter afferent sensory fibres in burning mouth syndrome. Imaging studies in patients have also suggested central nervous system changes (Jääskeläinen et al., 2001; Hagelberg et al., 2003).

More recently, an enticing hypothesis has been proposed that burning mouth syndrome is associated with an alteration of gonadal, adrenal and neuroactive steroid levels. Woda et al. suggested that chronic anxiety or stress results in a dysregulation of adrenal steroids, a reduction in 
adrenal steroids will, in turn, lead to an altered production of neuroactive steroids in skin, mucosa and the nervous system (Woda et al., 2009). The relationship with menopause is captured by the suggestion that the dramatic fall in gonadal steroids that occurs at that time further alters the production of neuroactive steroids. This "perfect storm" results in neurodegenerative alterations in small nerve fibres and areas of the brain related to somatic sensation. Thus explaining the peripheral and central changes already demonstrated in this condition. The article describing this concept in detail is followed by commentaries and is recommended reading (Zakrzewska, 2009; Eliav, 2009; El-Etr, 2009).

\section{Management}

The first thing the clinician must do is eliminate all potential local/systemic factors including smoking, medication (if feasible) and treat possible underlying conditions. The second and equally important is to reassure the patient that his/her complaint is real and not fatal. Antidepressants have long been considered the mainstay for the management of neuropathic pain disorders. An excellent evidence based review of the treatment modalities for burning mouth syndrome was reported by the Cochrane Collaboration (Zakrzewska et al., 2005). There is anecdotal evidence of some effectiveness for both tricyclic amines and selective serotonin reuptake inhibitors but this is poorly supported in controlled experiments. As well, the antidepressants have significant side effects such as somulence, dizziness, cardiac concerns and, interestingly enough, xerostomia. Both anecdotal and experimental reports have demonstrated symptomatic reduction with cognitive behavioral therapy (CBT) with the added benefit of no adverse effects (Bergdahl et al., 1995). Further improvement has been noted when cognitive behavioral therapy is combined with pharmacological management. Alpha-lipoic acid has been shown to result in effective symptom reduction, particularly when combined with cognitive behavioral therapy (Femiano and Scully, 2002; Femiano et al., 2004; Steele et al., 2008).

Anticonvulsants have become more popular lately for treatment of neuropathic pain disorders as well and hence have been tried for burning mouth syndrome. Gabapentin has demonstrated mixed results and studies are ongoing with pregabalin (White et al., 2004; Heckmann et al., 2006). Clonazepam reports and studies exhibit some promise (Gremeau-Richard et al., 2004). Thus far, it appears that low doses are required however somulence and addiction are potential adverse effects to consider.

Other modalities have also been considered. Systemic capsaicin has been shown to reduce pain however there is a significant incidence of adverse effects, particularly gastric pain (Petruzzi et al., 2004). Similarly topical steroid hormones and anti-inflammatory rinses have been tried with little evidence of effectiveness in reducing or eliminating the symptoms of burning mouth syndrome, particularly when compared to placebo or spontaneous remission rates (Pisanty et al., 1975; Sardella et al., 2006; Patton et al., 2007).

\section{Conclusions}

Available evidence suggests that burning mouth syndrome is a multifactorial disorder with a physiological basis.

Psychological factors are likely often operative but unlikely to be the primary cause.

Other oral diseases/disorders must be ruled out first.

Patient reassurance is paramount.

The most promising therapeutic approaches at present include some combination of CBT, alphalipoic acid and/or clonazepam.

Concurrent treatment of any significant psychiatric disorder should also be considered, if present.

This review was first presented in part at the AAOMS/CAOMS Annual Meeting on October 15, 2009 in Toronto, Canada.

\section{References}

Bergdahl J, Anneroth G, Perris H (1995). Cognitive therapy in the treatment of patients with resistant burning mouth syndrome: a controlled study. J Oral Pathol Med, 24(5): 213-215. 
El-Etr M (2009). Critical commentary 3: steroid dysregulation and stomatodynia (burning mouth syndrome). $J$ Orofacial Pain, 23(3): 216-218.

Eliav E, Nasri-Heir C (2009). Critical commentary 2: steroid dysregulation and stomatodynia (burning mouth syndrome). J Orofacial Pain, 23(3): 214-215.

Femiano F, Gombos F, Scully C (2004). Burning mouth syndrome: open trial of psychotherapy alone, medication with alpha-lipoic acid (thioctic acid), and combination therapy. Med Oral, 9(1): 8-13.

Femiano F, Scully C (2002). Burning mouth syndrome (BMS): double blind controlled study of alpha-lipoic acid (thioctic acid) therapy. J Oral Pathol Med, 31(5): 267-269.

Formaker BK, Mott AE, Frank ME (1998). The effects of topical anesthesia on oral burning in burning mouth syndrome. Ann NY Acad Sci, 855: 776-780.

Gao J, Chen L, Zhou J, Peng J (2009). A case-control study on etiological factors involved in patients with burning mouth syndrome. J Oral Pathol Med, 38(1): 24-28.

Gao S, Wang Y, Wang Z (2000). Assessment of trigeminal somatosensory evoked potentials in burning mouth syndrome. Chin J Dent Res, 3(1): 40-46.

Grémeau-Richard C, Woda A, Navez ML, Attal N, Bouhassira D, Gagnieu MC, et al. (2004). Topical clonazepam in stomatodynia: a randomized placebocontrolled study. Pain, 108(1/2): 51-57.

Hagelberg N, Fossell H, Aalto S, Luutonen S, Nagren K, Jääskeläinen S (2003). Striatal D1 and D2 receptors in burning mouth syndrome. Pain, 101(1/2): 149-154.

Headache Classification Committee of the International Headache Society (2004). Classification and diagnostic criteria for headache disorders, cranial neuralgias and facial pain. Cephalgia, 24(Suppl 1): 8-160.

Heckmann SM, Heckmann JG, Ungethüm A, Hujoel P, Hummel T (2006). Gabapentin has little or no effect in the treatment of burning mouth syndrome - results of an open label pilat study. Eur J Neurol, 13(7): e6-e7.

Jääskeläinen SK, Rinne JO, Forssell H, Tenovua O, Kaasinen V, Sonninen P, et al. (2001). Role of the dopaminergic system in chronic pain - a fluordopaPET study. Pain, 90(3): 257-260.

Lipton JA, Ship JA, Larach-Robinson D (1993). Estimated prevalence and distribution or reported orofacial pain in the United States. J Amer Dent Assoc, 124(10): 115-121.

Marino R, Capaccio P, Pignataro I, Spadari F (2009).
Burning mouth syndrome: the role of contact hypersensitivity. Oral Dis, 15(4): 255-258.

Oh R, Brown DL (2003). Vitamin $\mathrm{B}_{12}$ deficiency. Am Fam Physician, 67(5): 979-986.

Patton LL, Siegel MA, Benoliel R, De Laat A (2007). Management of burning mouth syndrome: systematic review and management recommendations. Oral Surg Oral Med Oral Pathol Oral Radiol Endod, 103(Suppl 1): 539.e1-539.e13.

Petruzzi M, Lauritano D, de Benedittis M, Baldoni M, Serpico R (2004). Systemic capsaicin for burning mouth syndrome: short-term results of a pilot study. $J$ Oral Pathol Med, 33(2): 111-114.

Pisanty S, Rafaely B, Polishuk WZ (1975). The effect of steroid hormones on buccal mucosa of menopausal women. Oral Surg Oral Med Oral Pathol, 40(3): 346353.

Sardella A, Lodi G, Demarosi F, Bez C, Cassano S, Carrassi A (2006). Burning mouth Syndrome: a retrospective study investigating spontaneous remission and response to treatments. Oral Dis, 12(2): 152-155.

Steele JC, Bruce AJ, Drage LA, Rogers RS (2008). Alpha-lipoic acid treatment of 31 patients with sore, burning mouth. Oral Dis, 14(6): 529-532.

Wessely S, Nimnuan C, Sharpe M (1999). Functional somatic syndromes: one or many? Lancet, 354(9182): 936-939.

White TL, Kent PF, Kurtz DB, Emko P (2004). Effectiveness of gabapentin for treatment of burning mouth syndrome. Arch Otolaryngol Head Neck Sug, 130(6): 786-788.

Woda A, Dao T, Grémeau-Richard C (2009). Steroid dysregulation and stomatodynia (burning mouth syndrome). J Orofacial Pain, 23(3): 202-210.

Yilmaz Z, Renton T, Yiangou Y, Zakrzewska J, Chessell IP, Bountra C, et al. (2007). Burning mouth syndrome as a trigeminal small fibre neuropathy: increased heat and capsaicin receptor TRPV1 in nerve fibres correlates with pain score. J Clin Neurosci, 14(9): 864-871.

Zakrzewska JM (1995). The burning mouth syndrome remains an enigma. Pain, 62(3): 253-257.

Zakrzewska JM (2009). Critical commentary 1: steroid dysregulation and stomatodynia (burning mouth syndrome). J Orofacial Pain, 23(3): 211-213.

Zakrzewska JM, Forssell H, Glenny AM (2005). Interventions for the treatment of burning mouth syndrome. Cochrane Database Syst Rev, (1): CD002779.

*Corresponding author: David Mock

Address: Faculty of Dentistry, University of Toronto, Toronto, Canada

Tel: 0014169794910 E-mail: david.mock@dentistry.utoronto.ca 